\title{
Apoyos que reciben estudiantes de secundaria con discapacidad en escuelas regulares: ¿Corresponden a lo que dicen las leyes?
}

\section{Educational Supports Received by Middle School Students with Disabilities in Regular Classrooms. Do they correspond to what the law says?}

\author{
Vasthi Jocabed Flores Barrera ${ }^{1}$ \\ Universidad Autónoma de San Luis Potosí \\ Facultad de Psicología \\ San Luis Potosí, México \\ vasthiflores@gmail.com \\ ORCID: orcid.org/0000-0002-1102-5479 \\ Ismael García Cedillo ${ }^{2}$ \\ Universidad Autónoma de San Luis Potosí \\ Facultad de Psicología \\ San Luis Potosí, México \\ ismaelgace@yahoo.com.mx \\ ORCID: orcid.org/0000-0002-4820-5633
}

Recibido: 6 setiembre 2014 Aceptado: 16 junio 2016 Corregido: 22 abril 2016

Resumen: En México existen leyes que garantizan los derechos de las personas con discapacidad en relación con su educación, las cuales mencionan apoyos de infraestructura, tecnológicos, didácticos, becas y de orientación y capacitación, entre otros. En el presente trabajo se busca identificar la relación entre los apoyos que, teóricamente, debe recibir el estudiantado con discapacidad y los que realmente recibe para garantizar su inclusión. Se hicieron dos estudios de caso con estudiantes de segundo grado de secundaria, uno con trastorno de Asperger y otro con discapacidad intelectual, a quienes se observó durante 13 horas de clase; se entrevistó a sus madres, docentes regulares, de educación especial y estudiantes con discapacidad y sin esta. Los resultados muestran que media mucha distancia entre los apoyos que deberían recibir y los que reciben, pues los únicos apoyos que reciben provienen de profesionales de educación especial, y son insuficientes para garantizar su inclusión.

Palabras clave: Integración educativa, secundaria pública, discapacidad intelectual, asperger, educación inclusiva.

Abstract: Mexican's laws guarantee educational rights of disabled people, including the right of infrastructure, technological and scholarship supports, orientation and training, among others. The

1 Licenciada en Psicología por la Universidad Autónoma de San Luis Potosí. Promotora comunitaria en Centro de Desarrollo Comunitario Rural Atlas.

2 Doctor en Psicología Clínica por la Universidad Nacional Autónoma de México. Cargos ocupados: Director del Proyecto Nacional de Integración Educativa (1995-2001); psicólogo clínico del Latin American Health Institute, de Bostos, Ma. (2002-2004). Profesor-investigador de la Universidad Autónoma de San Luis Potosí desde el 2004. Autor del Diplomado en Educación Inclusiva, que se imparte a nivel nacional desde 2009. 
objective of this research was to identify the relationship between the educative supports that disabled students should receive in middle school and those that they actually receive. Two case studies were conducted with second grade middle school students diagnosed with Asperger's Syndrome and intellectual disability respectively. The students were observed during 13 class hours. In addition, the mothers, regular and special education teachers, and disabled or non-disabled classmates were interviewed. The results show that the supports provided to middle school disabled students were minimal in relation with those they are supposed to receive. USAER provides the support, but it does not correspond to the inclusive model.

Key words: Educational integration, public middle school, intellectual disability, Asperger Syndrome, inclusive education, USAER.

\section{Introducción}

En el ámbito internacional, el proceso de educación inclusiva ha ganado preponderancia, al punto que se le considera un proyecto mucho más a mbicioso y deseable que el de la integración educativa (Blanco, 2000). Sin embargo, el significado y los alcances de la educación inclusiva dependen mucho de las condiciones locales en las que se pone en marcha (Romero y García, 2013). En México, la integración educativa consiste en la incorporación plena de estudiantes con necesidades educativas especiales con discapacidad y sin esta a las escuelas regulares, lo que implica diseñar e implementar adaptaciones curriculares para posibilitar mayores aprendizajes para la población integrada. El concepto de necesidades educativas especiales (NEE) está ligado al proceso de integración educativa, y no hace referencia a una condición o problema, sino a los requerimientos de apoyo derivados de la interacción del estudiantado con el ambiente escolar (Mares e Ito, 2005). Por su parte, la educación inclusiva (EI) se define como la participación y acceso a una educación de calidad de toda la niñez, sin importar su condición física, económica, social y cultural, para lo cual es necesaria la transformación del sistema educativo para que respalde y favorezca la búsqueda permanente de la calidad y el éxito educativo (UNESCO, UNICEF, Fundación Hineni, 2003).

En México, la EI es relativamente joven, apenas han pasado alrededor de quince años desde que se inició su implementación en las escuelas (García, Escalante, Escandón, Fernández, Mustri y Puga, 2003). Por lo mismo, se esperaría que generara un buen número de investigaciones para identificar sus condiciones, sus fortalezas y sus debilidades, pero estas son muy escasas. Un ejemplo de lo anterior es que no se encontró ninguna investigación publicada sobre el tema del presente estudio.

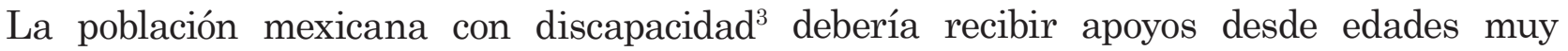
tempranas. Sin embargo, a pesar de lo que dice la Reglamento de la Ley General para la Inclusión de las Personas con Discapacidad (Gobierno Constitucional de los Estados Unidos Mexicanos , 2011), tales apoyos no se concretan, pues en los centros educativos no hay recursos tecnológicos, de infraestructura accesible y de vinculación con otras instituciones privadas o de educación superior que favorezcan al estudiantado integrado. Las capacitaciones que se ofertan

3 En el presente trabajo se trabajará con estudiantes con discapacidad porque la Ley de Inclusión de Personas con Discapacidad y su reglamentación mencionan que este grupo son quienes deben recibir los apoyos, no se habla de apoyos a estudiantes con NEE. 
al personal docente no parecen ser las adecuadas para mejorar su práctica docente e identificar los apoyos que esta población estudiantil precisa de él (Romero y García, 2013). Como se dijo antes, no se ha encontrado ningún estudio que documente si los apoyos que requiere el alumnado realmente se le está entregando. Por ejemplo, no hay evidencias de que se otorguen becas para educación de manera generalizada a la población con discapacidad o, en el caso de la población de mayor edad, para su capacitación. El objetivo de la presente investigación es identificar la coincidencia entre los apoyos que teóricamente deben otorgársele a la población estudiantil con discapacidad y los que realmente recibe, en el nivel de secundaria. Esta identificación es muy necesaria porque la adecuada integración del estudiantado depende, en buena medida, de los recursos que se pongan a su alcance.

\section{Marco Teórico}

\section{Breve historia}

El recorrido histórico de la educación especial (EE) en México empieza a mediados del siglo XIX, cuando se crea la Escuela Nacional de Ciegos y después la Escuela Nacional de Sordos. Gradualmente se crearon algunas escuelas de EE para atender otras discapacidades y se abrieron distintas escuelas en todos los estados del país (Dirección de Educación Especial del Distrito Federal, 2010). No fue sino hasta 1970 cuando se creó la Dirección General de Educación Especial, que trajo consigo un incremento muy notable en el número de escuelas especiales en todo el país (García, 2009). Con este hecho se empieza a notar el interés del gobierno por atender a la población con discapacidad, pero no fue sino hasta la década de los noventa cuando se le dio un impulso más decidido. Gracias al Acuerdo Nacional para la Modernización de la Educación Básica (1992) por sus siglas ANMEB, se consiguió la reforma a la Ley General de Educación, particularmente al Artículo 41 donde, entre otros aspectos, se estipula lo siguiente: "La educación especial está destinada a personas con discapacidad, transitoria o definitiva, así como a aquéllas con aptitudes sobresalientes" (Gobierno Constitucional de los Estados Unidos Mexicanos., 2015, p.18). Además de mencionar que la EE propiciaría la integración de estudiantes con discapacidad a los planteles de educación básica regular, en dicho artículo se estipuló que se ofrecería apoyo y orientación a los padres y madres de familia y al personal docente.

El ANMEB propició cambios drásticos en la EE, los cuales a su vez repercutieron en la educación básica (Jacobo et al., 2005). Dichos cambios buscaban cumplir con el deber del Estado de proporcionar una educación justa a todo el alumnado, garantizando la equidad. Así, los cambios en la organización y las funciones de EE tenían como finalidad promover la integración educativa, pues en ese tiempo la educación pública mantenía la segregación de las personas con discapacidad, que eran escolarizadas en lo que entonces eran las escuelas de EE (SEP, 2006).

Antes del ANMEB, la educación especial estaba organizada en servicios indispensables (centro de intervención temprana, escuelas de educación especial, centros de capacitación de educación especial, grupos integrados B para estudiantes con deficiencia mental leve y grupos integrados para personas hipoacúsicas en las escuelas regulares), y servicios complementarios 
(centros psicopedagógicos y grupos integrados $\mathrm{A}$, que atendían estudiantes en escuelas regulares con problemas de aprendizaje, aprovechamiento escolar, lenguaje y conducta), ofreciendo una atención sustentada en el modelo médico, con currículos distintos y específicos por discapacidad (SEP, 2006).

\section{Organización actual de la integración educativa en México}

Gracias al ANMEB, los servicios indispensables pasaron a convertirse en los actuales centros de atención múltiple (CAM), que funcionan con los mismos planes y programas de estudio de la educación básica y atienden a estudiantes con distintas discapacidades en grupos constituidos de acuerdo con su edad, sin importar la discapacidad que presenten (García, Romero, Motilla y Zapata, 2009). Los servicios complementarios pasaron a ser las unidades de servicio de apoyo a la educación regular (USAER), con la principal diferencia de que ahora es el profesorado de EE quien acude a las escuelas regulares a brindar asesoría a docentes y apoyo a las familias del alumnado con alguna NEE. Así, el profesorado de USAER pasó de trabajar de manera casi autónoma a constituirse en un apoyo del centro educativo regular (García, 2007). La población que atiende el personal docente de EE se amplió y diversificó, ya que el concepto de NEE hace alusión a una población estudiantil que requiere de apoyos para su óptimo desarrollo escolar debido a factores relacionados con el contexto familiar, escolar y social (SEP, 2006).

En 1998 se creó el Proyecto Nacional de Integración Educativa, el cual implementó la IE en el país de manera gradual, ordenada y sistemática; en 2002 se transformó en el Programa Nacional para el Fortalecimiento de Educación Especial y la Integración Educativa (PNFEEIE). El PNFEEIE tuvo como objetivo responder a las demandas de una educación de calidad para todo el estudiantado, garantizando el acceso, la permanencia, la participación y el logro de aprendizajes principalmente de estudiantes con discapacidad, aptitudes sobresalientes y talentos específicos; buscó, también, consolidar una cultura de integración con miras a una sociedad inclusiva que brinde las mismas oportunidades a toda la ciudadanía (Secretaría de Educación Pública (SEP), 2002). Este programa describe las características que debe reunir la IE: a) que la población estudiantil con NEE estudie en escuelas y aulas regulares; b) que se le ofrezcan los apoyos que requiera, sobre todo en adecuaciones curriculares y c) que los centros educativos cuenten con el apoyo de personal de educación especial (Secretaría de Educación Pública (SEP), 2002).

Con el PNEEIE se intentaba que todos los niños y niñas tuvieran acceso a una educación de calidad, pasando del modelo médico al modelo educativo, lo cual implicaba atender no solo a las discapacidades, sino a las NEE; lo anterior también implicaba la comunicación y el trabajo colaborativo entre el personal de escuelas regulares y de educación especial, para de esta manera beneficiar también al grupo de estudiantes sin NEE (García, 2007). A finales de 2013, el PNEEIE desapareció y sus funciones se integraron al Programa para la Inclusión y Equidad Educativa, del cual a la fecha no se han publicado sus fundamentos.

\section{Referentes conceptuales}

La educación inclusiva responde a un derecho humano y constitutivo que tienen las personas con discapacidad (PcD), el cual está respaldado en tratados y acuerdos 
internacionales como la Convención de los Derechos de las Personas con Discapacidad promulgada en diciembre del 2006 en la Asamblea de la ONU; México fue de los primeros países en firmarla, para que en mayo del 2008 entrara en vigor en el país. El artículo 24 de este documento señala que los Estados deben asegurar que, en todos los niveles educativos, se brinde atención inclusiva, garantizando a las PcD el acceso a la educación en igualdad de condiciones y en la comunidad en la que vivan. De igual modo, los Estados deben brindar las posibilidades de aprender habilidades para la vida, propiciar la participación plena y el desarrollo social y académico (ONU, 2006).

Dentro del marco legal de México, la Ley General para la Inclusión de las Personas con Discapacidad promulgada en mayo del 2011, en el capítulo tres, artículo 12, señala que es la Secretaría de Educación Pública la encargada de promover el derecho a la educación de las PcD a través del soporte técnico-pedagógico y apoyos didácticos. Es decir, que se debe equipar a los planteles educativos de manera que sean accesibles para las personas con discapacidad; menciona que el profesorado que intervenga directamente en la integración debe recibir capacitación y actualización sobre el tema. En el párrafo VI, señala que al grupo de estudiantes con discapacidad se le debe proveer de ayudas técnicas que apoyen su rendimiento académico a través de materiales didácticos, equipos computarizados y todos aquellos recursos necesarios para brindar una educación de calidad. Aunado a lo anterior, en el párrafo VII se menciona que se debe establecer un programa nacional de becas educativas y de capacitación para las personas con discapacidad. El capítulo concluye con el artículo 15, que estipula que el objeto de la EE es la formación para la vida independiente, permitiendo a las personas tener un desempeño académico equitativo y, con esto, evitar el rezago o la discriminación (Ley General para la Inclusión de las Personas con Discapacidad, 2011).

En el Reglamento de dicha ley, en el artículo 12 se estipula que la Secretaría de Salud impulsará la creación de bancos de órtesis, prótesis, ayudas técnicas y de medicinas de uso restringido, a los que podrán acceder las personas que presenten alguna discapacidad; en el artículo 35 se hace mención específica del procedimiento que se deberá seguir para otorgar las becas educativas a las PcD; asimismo, señala que las ayudas técnicas "deberán diseñarse y producirse en medios audiovisuales, informáticos, multimedia y otros recursos tecnológicos con el propósito de que los mismos sean accesibles para los estudiantes con discapacidad" (Gobierno Constitucional de los Estados Unidos Mexicanos, 2012, p. 7). Incluso hace mención de que se podrán crear lazos entre instituciones de educación superior a fin de garantizar el cumplimiento de estos apartados y ampliar las oportunidades de participación de las PcD.

Por su parte, la Secretaría de Educación Pública (SEP) destaca que el profesorado de EE es factor clave para el logro de la integración educativa del alumnado en situación de vulnerabilidad y para la mejora de las prácticas docentes en las escuelas regulares (Comisión Nacional SEP-SNTE de Carrera Magisterial, 2011).

La SEP ofreció capacitaciones al cuerpo docente en servicio, en las cuales promovió lo siguiente:

- La aceptación de la integración educativa, a partir de considerar que la escuela regular es el mejor lugar para el desarrollo óptimo de los niños y las niñas.

- La consideración de que la integración de estudiantes con discapacidad o NEE 
representa una oportunidad de beneficiarse para todo el grupo, gracias a la construcción de cultura y políticas de inclusión.

- La noción de que la función del personal docente frente al grupo es ajustar sus prácticas para el beneficio de la comunidad estudiantil, así como hacer adecuaciones curriculares (Comisión Nacional SEP-SNTE de Carrera Magisterial, 2011).

Además, la SEP puso mayor énfasis en el trabajo colaborativo entre el personal docente de escuela regular y de EE, quienes deberían colaborar en la detección, intervención y seguimiento de estudiantes con NEE para favorecer no solo al estudiante, sino al centro educativo en general (García, Escalante, Escandón, Fernández, Mustri y Puga, 2000).

El apoyo directo a la integración educativa, como se mencionó antes, lo proporcionan las USAER, las cuales están conformadas por un director o directora, profesionistas del área de psicología, comunicación, profesionales de trabajo social y educación especial. Atienden a un promedio de cinco escuelas de manera itinerante, con excepción del personal de educación especial llamado comúnmente maestras o maestros de apoyo, que permanecen en una escuela; tienen entre sus funciones "desarrollar y poner a disposición de la educación básica recursos humanos, conceptuales y metodológicos para la mejora de los procesos de enseñanza y de aprendizaje" (SEP, 2009, p. 7). De esta manera, es responsabilidad conjunta del personal docente de las escuelas regulares y profesionistas de las USAER obtener los mejores resultados posibles en la educación del alumnado con NEE. El apoyo que debe brindar la USAER a las escuelas regulares va desde la elaboración del plan anual de trabajo, la asesoría a docentes y a padres y madres de familia y, en última instancia, el trabajo con la población con NEE (se menciona que la intervención deberá de ser dentro del aula) (SEP, 2009).

Ahora bien, las evaluaciones que se han hecho a la integración del estudiantado con NEE indican que el personal docente conoce y está familiarizado con los principales conceptos de NEE y la integración educativa (SEP, 2002); no obstante, estos logros no son suficientes para impactar al centro educativo y que se reflejen en las prácticas inclusivas de todo el personal docente (Jacobo, s. f.). En relación con las evaluaciones psicopedagógicas y adecuaciones curriculares, el profesorado expresa no tener las herramientas necesarias para implementarlas, aunque acepta que la atención a las NEE requiere flexibilidad curricular y hacer uso de una diversidad de estrategias para favorecer el aprendizaje no solo de personas con discapacidad o aptitudes sobresalientes, sino en general del estudiantado (SEP, 2002). Jacobo (s. f.) menciona que el personal docente de USAER carece de conocimientos para atender a estudiantes con discapacidad severa, quienes requieren de más recursos y materiales, por lo que se quedan sin que se resuelvan sus necesidades en las escuelas regulares.

Recapitulando; los apoyos que debería recibir el estudiantado con NEE asociadas a discapacidad en las escuelas regulares de educación básica son las que aparecen en la figura 1. 
Obligación del Estado

De parte de las escuelas regulares

- Para la institución:

Infraestructura accesible con condiciones del diseño universal.

- Para la comunidad estudiantil: Becas educativas y de capacitación, apoyos tecnológicos, soportes técnico-pedagógicos $\mathrm{y}$ didácticos.
Obligación de USAER

De parte de educación especial

- Para la institución:

Asesoría para elaboración

de plan anual o planeación estratégica.

- Para la comunidad estudiantil: Evaluación psicopedagógica y propuesta curricular adaptada. Seguimiento y apoyo hasta eliminar o minimizar las barreras para el aprendizaje y participación escolar.

Figura 1. Apoyos que debería recibir la población estudiantil con discapacidad en México. Esquema elaborado para la investigación con base en los diferentes documentos normativos.

En el presente estudio se trabajó con dos estudiantes de género masculino que reciben apoyo de la USAER en la secundaria a la que asisten. Los alumnos están diagnosticados con discapacidad intelectual y síndrome de Asperger (trastorno del espectro autista), respectivamente. En la actualidad, para arribar a estos diagnósticos suelen utilizarse los criterios que señala la Asociación Americana de Psiquiatría en el DSM-IV-TR o los criterios de la Organización Mundial de la Salud en el CIE-10 (Muñoz y Portillo, 2007), aunque a la fecha de la investigación ya circulan versiones actualizadas que tal vez empiecen a utilizarse con mayor frecuencia.

La discapacidad intelectual se entiende como la limitación significativa en el funcionamiento intelectual y la conducta adaptativa de tipo conceptual, social y práctica, originada antes de los 18 años (García, 2005). El síndrome de Asperger se define como un trastorno generalizado del desarrollo, el cual se manifiesta en tres ámbitos: interacción social, comunicación y habilidades o capacidades de imaginación (Arape y Pierre, 2010).

Para esta investigación se definió el siguiente objetivo general: Identificar la coincidencia entre los apoyos que teóricamente debe recibir el estudiantado con discapacidad en el nivel de secundaria y los que realmente recibe. Además, se buscaron los siguientes objetivos específicos:

- Identificar las principales necesidades prácticas, metodológicas y de recursos de la población estudiantil, que deben ser cubiertas por el Estado.

- Detectar las principales necesidades prácticas, metodológicas y de recursos de quienes participan, que deben ser cubiertas por la secundaria a la que asisten (C) y (E). 
- Identificar las principales necesidades prácticas, metodológicas y de recursos del estudiantado, que deben ser cubiertas por el Servicio de Educación Especial.

- Detectar las principales necesidades prácticas, metodológicas y de recursos del estudiantado que deben ser cubiertas por sus padres, madres o personas tutoras.

Se trata de un estudio de casos cualitativo, descriptivo, transversal y de campo. Se trabajó con dos estudiantes de género masculino, ambos con discapacidad, seleccionados de manera no probabilística, por conveniencia. Los alumnos asisten a dos escuelas secundarias públicas del Estado de San Luis Potosí. Ambos están en segundo grado, pertenecen al "grupo de apoyo" y son atendidos por el área de psicología y comunicación.

En el primer caso (E), se trata de un alumno de 13 años, su familia nuclear está compuesta por padre, madre y tres hermanos mayores que él. Su condición socioeconómica es media alta. En 2010 fue diagnosticado con síndrome de Asperger por un psicólogo particular y en 2013 el diagnóstico fue corroborado por un hospital psiquiátrico infantil en la ciudad de México.

El segundo caso (C) tiene 16 años de edad, pertenece a una familia uniparental, de condición socioeconómica media; tiene dos hermanos menores que él. En el 2005 le realizaron estudios de neurología, la conclusión del médico fue que "funcionaba dentro de los límites normales para la edad" pero le recetó Metilfenidato (Ritalín). En 2012, también en un hospital público en la ciudad de San Luis Potosí, se le diagnosticó discapacidad intelectual al valorar los antecedentes médicos, de atención multidisciplinaria y por los resultados de las pruebas psicométricas.

Para obtener la información sobre algunos de los apoyos que reciben estos estudiantes se utilizó el formato de observación en aula (Metz, 2011); este formato ha sido utilizado en México en investigaciones previas relacionadas con la educación inclusiva y las prácticas docentes; es estructurado y permite obtener información sobre la distribución física del aula, comportamiento y número de estudiantes, recursos disponibles y estrategias de enseñanza. A la par, se utilizó una rúbrica de observación que incluye preguntas guía para la observación directa de estudiantes, del grupo y de docentes.

Se realizó una entrevista a los estudiantes con discapacidad, madres de familia y profesionistas de educación especial y regular, para saber los apoyos que ofrecen y la percepción que tienen los agentes involucrados sobre la inclusión en nivel secundaria, para contrastar la teoría con la práctica real. Los formatos de las entrevistas han sido utilizados previamente en investigaciones sobre inclusión (García y Romero, s. f.).

Para el acceso a las instituciones educativas se solicitó el permiso a sus directores y al director de la USAER que atiende a ambas escuelas. Para la recolección de la información se observó directamente a los participantes durante 13 horas clase y los recesos correspondientes a una semana. Se realizaron las entrevistas, durante las cuales a las madres se les planteó el objetivo del estudio y accedieron a su participación. Dicha solicitud también se hizo al resto de

4 Aunque muchos autores y autoras están en contra de usar esta etiqueta con la población estudiantil que recibe apoyo de EE, es muy común que así se les nombre en los centros educativos (Romero y García, 2013). 
las personas participantes. Para las entrevistas se solicitaron espacios y tiempos disponibles de cada participante, accediendo todas las personas con buena disposición.

La mayoría de las observaciones se hicieron dentro del salón de clase. En el caso de (E), además, se hicieron en el laboratorio del taller de ofimática. Se realizaron un total de ocho observaciones, las materias observadas fueron: inglés, historia, matemáticas, taller de ofimática y ciencias. En el caso de (C) se realizaron cinco observaciones, ya que dos días el alumno no asistió a la escuela y hubo suspensión de clases. Se observaron las materias de artes, ciencias, inglés, historia y formación cívica y ética. Para el presente artículo se utilizó la información pertinente para la comparación de los apoyos que reciben y deberían recibir los estudiantes seleccionados; de las entrevistas solo se tomaron los apartados de información personal y las percepciones sobre los apoyos a la integración educativa, lo mismo para las observaciones directas a los estudiantes.

Después de analizar los datos obtenidos de las observaciones, entrevistas y expedientes psicopedagógicos, se concentró la información en tablas para comparar los apoyos que teóricamente debe recibir la población con discapacidad inscrita en escuelas regulares con los apoyos que realmente recibe.

En la tabla 1 se señalan los apoyos que recibe (E) y el nivel de satisfacción según las observaciones en el aula y las entrevistas a docentes y a su madre. Las categorías de la tabla se tomaron de la teoría, esto es, de las diferentes leyes y reglamentaciones que definen los apoyos que deben darse a las PcD. Las casillas en blanco son los apoyos que recibe satisfactoriamente, las casillas sombreadas de gris tenue son los apoyos que recibe parcialmente y las casillas sombreadas de gris obscuro y letras blancas son los que no recibe (tabla 1).

Tabla 1

Apoyos que recibe (E)

\begin{tabular}{|c|c|c|c|}
\hline & \multicolumn{3}{|c|}{ APOYOS QUE DEBERÍAN RECIBIR LAS PcD } \\
\hline & En la institución & Para el estudiantado & $\begin{array}{c}\text { Para el personal docente } y \\
\text { familias }\end{array}$ \\
\hline \multirow{3}{*}{$\begin{array}{l}\text { De parte del } \\
\text { Estado y de } \\
\text { las escuelas } \\
\text { regulares }\end{array}$} & \multirow{3}{*}{$\begin{array}{c}\text { Infraestructura accesible } \\
\text { bajo condiciones de diseño } \\
\text { universal }\end{array}$} & $\begin{array}{l}\text { Becas educativas y de } \\
\text { capacitación }\end{array}$ & Capacitación y actualización \\
\hline & & Apoyos tecnológicos & \\
\hline & & $\begin{array}{c}\text { Soportes técnico-pedagógicos y } \\
\text { didácticos }\end{array}$ & $\begin{array}{l}\text { Sensibilización sobre } \\
\text { educación inclusiva }\end{array}$ \\
\hline \multirow{3}{*}{$\begin{array}{l}\text { De parte de } \\
\text { Educación } \\
\text { Especial }\end{array}$} & \multirow{3}{*}{$\begin{array}{c}\text { Asesoría para } \\
\text { elaboración del plan } \\
\text { anual o planeación } \\
\text { estratégica }\end{array}$} & Evaluación psicopedagógica & $\begin{array}{l}\text { Orientación y estrategias } \\
\text { multidisciplinarias }\end{array}$ \\
\hline & & $\begin{array}{c}\text { Propuesta curricular } \\
\text { adaptada }\end{array}$ & $\begin{array}{c}\text { Acciones a favor de sensibilización } \\
\text { y toma de conciencia }\end{array}$ \\
\hline & & $\begin{array}{c}\text { Seguimiento y apoyo hasta } \\
\text { eliminar o minimizar las } \\
\text { barreras para el aprendizaje y } \\
\text { participación escolar }\end{array}$ & $\begin{array}{l}\text { Apoyo colaborativo entre personal } \\
\text { de educación regular (ER) y EE }\end{array}$ \\
\hline
\end{tabular}

Nota: Elaboración propia. 
La mayoría de los apoyos que recibe (E) son parciales, es decir, no son constantes y efectivos, sino se hacen intentos a fin de promover la integración y el aprendizaje del estudiante, pero no son evaluados de manera regular y no hay retroalimentación para la toma de decisiones.

En cuanto a la infraestructura de la institución, solo hay rampas de acceso, la mayoría sin las condiciones de diseño universal, no hay baños adaptados, mobiliario ergonómico ni accesible, tampoco adecuaciones para estudiantes con discapacidades sensoriales.

El personal de EE colabora con el centro educativo en la toma de decisiones a favor de la integración mediante su participación en el Consejo Técnico Escolar, donde las maestras de apoyo y algún profesional del equipo multidisciplinario exponen las necesidades que detectan en su área y participan con opiniones y sugerencias durante las sesiones, pero no hay trabajo colaborativo para el diseño del plan de trabajo anual de la escuela.

(E) nunca ha recibido un apoyo de beca para su educación o capacitación por parte del Estado, a pesar de que además de la escuela acude a actividades extra curriculares de arte (piano) y deporte (natación), financiadas por su familia.

En relación con el apoyo tecnológico, (E) no parece necesitarlo; aunque está en el taller de ofimática no hay recursos tecnológicos que lo beneficien a la par que al resto del alumnado. En cuanto a los soportes técnico pedagógicos, solo se han implementado algunas estrategias pedagógicas con (E), como el uso de agenda de tareas, compañera monitora, revisión de trabajos y libretas, entre otras, pero no se proporcionan apoyos técnicos.

De acuerdo con las entrevistas al personal docente, en el apartado sobre la capacitación que han recibido, se encontró que los cursos fueron intensivos, teóricos e impartidos por docentes comisionados que, si bien recibieron la capacitación como instructores, no mostraron el domino necesario del tema, por lo que la dinámica y secuencia de los cursos se basaron en teorías y conceptos ajenos a la práctica docente. Por su parte, la mayoría acudió a los cursos solo como requisito administrativo.

El trabajo multidisciplinario es poco frecuente, ya que la mayoría del grupo profesional de EE se enfoca al trabajo individual o por área, lo que hace que el tiempo y los recursos sean limitados y dirigidos principalmente al "grupo de apoyo".

Por lo anterior, se considera que el trabajo colaborativo entre el personal de EE y ER es parcial, ya que la mayoría de las veces el acercamiento entre ambos es en reuniones de academia o del plantel y las estrategias son generales, a excepción de algunos casos de estudiantes en específico y situaciones emergentes, donde el cuerpo docente comparte información y sugerencias.

Los únicos apoyos que recibe (E) satisfactoriamente están relacionados con algunos aspectos del trabajo del equipo de USAER, es decir, la evaluación psicopedagógica, propuesta curricular adaptada y el seguimiento, los cuales se encuentran a manera de informes incorporados al expediente del alumno. Se tienen formatos que registran la información personal del estudiante, los antecedentes familiares, escolares y médicos, los instrumentos utilizados para la valoración, los resultados, las conclusiones y sugerencias y las acciones a favor del estudiante, así como los reportes de seguimiento del área de psicología, comunicación y maestra de apoyo. (E) tiene propuesta curricular adaptada desde noviembre del 2012 y 
actualizada para el ciclo escolar 2013-2014, donde se resaltan adecuaciones metodológicas sugeridas al personal docente de la escuela regular, por ejemplo que (E) se ubique frente y cerca del docente, que el profesorado sea flexible para recibir las tareas o trabajos después de la fecha general, de parte de la maestra de apoyo el seguimiento de la agenda de tareas.

En la tabla 2 se presentan los resultados del alumno (C).

Tabla 2

Apoyos que recibe (C)

\section{Apoyos que deberían recibir las PcD}

En la institución

\begin{tabular}{|c|c|c|c|}
\hline \multirow[b]{2}{*}{$\begin{array}{l}\text { De parte del } \\
\text { Estado y de } \\
\text { las escuelas } \\
\text { regulares }\end{array}$} & \multirow[b]{2}{*}{$\begin{array}{c}\text { Infraestructura accesible } \\
\text { bajo condiciones de diseño } \\
\text { universal }\end{array}$} & $\begin{array}{c}\text { Becas educativas y de } \\
\text { capacitación }\end{array}$ & Capacitación y actualización \\
\hline & & $\begin{array}{c}\text { Soportes técnico-pedagógicos y } \\
\text { didácticos }\end{array}$ & $\begin{array}{l}\text { Sensibilización sobre } \\
\text { educación inclusiva }\end{array}$ \\
\hline \multirow{3}{*}{$\begin{array}{l}\text { De parte de } \\
\text { Educación } \\
\text { Especial }\end{array}$} & \multirow{3}{*}{$\begin{array}{c}\text { Asesoría para elaboración } \\
\text { del plan anual o planeación } \\
\text { estratégica }\end{array}$} & Evaluación psicopedagógica & $\begin{array}{l}\text { Orientación y estrategias } \\
\text { multidisciplinarias }\end{array}$ \\
\hline & & $\begin{array}{c}\text { Propuesta curricular } \\
\text { adaptada }\end{array}$ & $\begin{array}{c}\text { Acciones a favor de } \\
\text { sensibilización y toma de } \\
\text { conciencia }\end{array}$ \\
\hline & & $\begin{array}{c}\text { Seguimiento y apoyo hasta } \\
\text { eliminar o minimizar las } \\
\text { barreras para el aprendizaje y } \\
\text { participación escolar }\end{array}$ & $\begin{array}{l}\text { Apoyo colaborativo entre } \\
\text { personal de ER y EE }\end{array}$ \\
\hline
\end{tabular}

Nota: Elaboración propia.

En la tabla 2 se observa que (C) recibe menos apoyos que (E) y los que recibe, en su mayoría, son parciales. Al igual que en el caso anterior, el apoyo que recibe satisfactoriamente proviene del servicio de EE, la evaluación psicopedagógica se encuentra incorporada al expediente del alumno y contiene datos de identificación, antecedentes familiares, médicos y escolares, así como las evaluaciones iniciales, las acciones tomadas respecto a los resultados y anotaciones del área de psicología y lenguaje.

Los apoyos parciales indican que, si bien se han hecho intentos por satisfacer las necesidades de (C), no se ha logrado el objetivo de beneficiarlo en sus aprendizajes y desempeño escolar, pues son muy deficientes. En cuanto a los apoyos a la institución, las adecuaciones en infraestructura son mínimas, algunas rampas y edificios intercomunicados, los cuales fueron 
construidos recientemente, pero las áreas de recreación, sanitarias, los laboratorios y aulas carecen de infraestructura adecuada para integrar al grupo estudiantil con discapacidades sensoriales o motrices. No hay asesoría de parte de Educación Especial hacia la institución para la planeación anual donde se tomen medidas a favor de la inclusión y respecto al alumno.

Se encontró que (C) nunca ha recibido becas para su educación y capacitación. No ha recibido suficientes apoyos tecnológicos ni soportes técnico-pedagógicos. Tampoco tiene propuesta curricular adaptada.

Los apoyos que recibe directamente (C) en cuanto a seguimiento también son parciales, ya que si bien se han tomado decisiones con la intención de favorecerlo, la realidad es que los resultados obtenidos no parecen ser exitosos, lo que se refleja en que las calificaciones que obtiene no corresponden a lo que realmente es capaz de hacer, es decir, (C) está lejos de cumplir con el perfil académico esperado de un estudiante de segundo grado de secundaria; su nivel de adaptación al ambiente se ha incrementado, pero con el resto del grupo no mantiene una relación de ayuda mutua y amistad y, por el contrario, ha imitado conductas inadecuadas.

En cuanto a los apoyos al personal docente, al igual que en el caso de (E), también recibió capacitación, pero con la misma dinámica, es decir, cursos teóricos ajenos a su práctica cotidiana. El personal docente que interactúa con $(\mathrm{C})$ no ha recibido sensibilización sobre inclusión, ya que, como se comentó antes, los cursos, diplomados o posgrados que cursa se enfocan al conocimiento teórico.

De parte de la USAER, las estrategias multidisciplinarias que se trabajan para (C) son de modalidad individual, por lo que no se logra unificar criterios entre el personal académico que lo atiende. Las acciones a favor de la concientización y toma de decisiones son principalmente hacia la familia y, en menor medida, hacia el personal docente de la secundaria. Por último, el apoyo colaborativo entre personal de EE y ER se produce solamente en reuniones generales o en casos específicos, pero no hay análisis de caso constantes o juntas de colegiado para analizar la situación de (C). En ambos casos el cuerpo docente de ER comentó respecto al apoyo al grupo de estudiantes integrados, ser más "tolerantes", aceptar los trabajos aún fuera de la fecha oficial de entrega, calificar según su esfuerzo y comportamiento dentro del salón. Y en comparación con el resto, también reportaron mayor comunicación con el profesorado de ER y EE para favorecer a este grupo; aunque, como se dijo antes, esto sucede esporádicamente.

En las entrevistas con las madres de ambos alumnos se encontró que su preocupación gira en torno a tres aspectos. El primero y más inquietante para ambas es sobre la continuidad de los estudios de sus hijos: reconocen que han recibido apoyo y orientación por parte de EE, pero temen el proceso de ingreso a nivel medio superior o, en su caso, al ámbito laboral. Expresaron sentirse temerosas de tomar la decisión incorrecta con respecto al futuro de sus hijos, ya que podrían exponerlos a ambientes y exigencias que sobrepasen sus capacidades y habilidades o, por el contrario, limitarlos de lo que esperan sea "su despegue". El segundo motivo de preocupación es su integridad personal, temen que sufran acoso escolar, agresión física o psicológica e incluso ataques sexuales. Y el tercer aspecto que preocupa a las madres es sobre la necesidad de que sus hijos logren una total autonomía e independencia, ya que ambas piensan que sus hijos aún no están preparados para valerse por sí mismos en caso de que ellas falten. 
Durante las observaciones directas a los alumnos se encontró que, en el ámbito escolar, ambos están significativamente distantes de sus responsabilidades mínimas, es decir, en el caso de (E), de no ser por el apoyo de sus compañeros y compañeras, perdería información e instrucciones importantes para la realización de las actividades durante la clase y para el trabajo en casa; en el caso de (C), frecuentemente está pasivo en clase, su atención y cumplimiento de las actividades durante el desarrollo de las materias son mínimos, lo que le lleva a cumplir insatisfactoriamente con sus trabajos en clase, en sus tareas en casa, ejercicios en el libro o libreta, trabajos en equipo, etcétera. En las entrevistas con los estudiantes se encontró que no logran establecer amistades en su grupo, solo reconocen a algunos compañeros con los que socializan superficialmente, la percepción que tienen de la escuela únicamente se relaciona con ejercicios, actividades en libros o libretas y la revisión de trabajos; esta percepción no trasciende al desarrollo de habilidades para el logro de autonomía y preparación profesional. Tienen una percepción positiva del profesorado, pues lo relacionan con la asignación de los trabajos escolares, les revisa las libretas y da las clases. Reconocen que reciben ayuda, pero también piden más de parte de su grupo para aligerar su permanencia en la clase y como herramienta de comprensión de actividades o tareas, pues prefieren acercarse a sus compañeros y compañeras que a los maestros. La ayuda que les gustaría recibir se relaciona con el acceso a una mayor variedad de actividades, pues suelen aburrirse o incomodarse con la rutina de los maestros y quisieran enfrentar actividades diferentes para aprender.

Por parte del Estado (E) necesita apoyos tecnológicos que promuevan su participación y aprovechamiento escolar. Es necesario que el Estado promueva un cambio de paradigma dentro de los centros escolares, a fin de que se valoren las virtudes y habilidades de los estudiantes en lugar de destacar sus deficiencias. La infraestructura es importante no solo para favorecer el acceso de estudiantes con discapacidad, sino para asegurar su permanencia. En el caso de (C), es necesario que cuente con material didáctico que facilite la comprensión de contenidos. Una necesidad importante en ambos estudiantes es contar con apoyos para actividades extracurriculares, las cuales, además de interesarles, les beneficia en lo académico y en el desarrollo de habilidades sociales y artísticas.

Ambos estudiantes necesitan de parte del Estado la garantía de atención médica especializada que pueda ofrecer un diagnóstico certero y un tratamiento efectivo, ya que en el caso de (C) la valoración diagnóstica parece carecer de información útil y clara para la madre, el alumno y el personal docente: el procedimiento para la valoración y diagnóstico parece desarticulado e improvisado. En el caso de (E), por el procedimiento lento, esporádico y poco confiable que ofrecen los servicios de salud locales, los padres de familia acudieron a servicios privados y foráneos.

En relación con sus docentes, (E) necesita no solo que sean flexibles e implementen las adecuaciones curriculares que sugiere el personal de $\mathrm{EE}$, sino que propongan y valoren los cambios metodológicos que le favorezcan a él y a todo el grupo. En el caso de (C), necesita que su secundaria informe sobre las adecuaciones curriculares que deben implementarse para favorecer el aprendizaje del alumno, con la finalidad de que todo el personal esté enterado y se trabaje bajo un mismo enfoque: necesitan más información sobre las capacidades y habilidades de (C) para no omitir o pasar por alto conductas de apatía o desinterés y confundirlo con falta de capacidad del alumno. 
Con respecto a su familia, (E) recibe apoyo no solo para actividades propias de su formación académica sino que le costean sus actividades extraescolares y la tecnología que necesita, aspectos importantes que le han favorecido en su proceso de aprendizaje. No obstante, necesita mayor coherencia entre lo que hace en casa (trabajos, tareas, investigaciones, repasos) y los resultados que obtiene directamente de su esfuerzo, dedicación y empeño, es decir, necesita que su trabajo refleje realmente lo que sabe y hace y no solo lo que debe cumplir, para que en el contexto escolar pueda desenvolverse con estas herramientas y no quede limitado a trabajos en casa entregados de manera extemporánea. En el caso de (C), sus necesidades son mayores, ya que no hay vinculación de la casa con la escuela, se delega la responsabilidad total al alumno y hay poca información sobre su desempeño o asistencia. Por ejemplo, en casa parece que se infiere que, como no hay reportes o citatorios por parte de la escuela todo está bien, por eso (C) precisa mayores apoyos relacionados a monitoreo y comunicación entre la escuela y el hogar, necesita trabajo de repaso en casa, desarrollar hábitos que le permitan incrementar autonomía y responsabilidad. También requiere el apoyo para la práctica de alguna actividad complementaria que favorezca su proceso de aprendizaje.

\section{Discusión}

Esta investigación permite conocer cuáles son los apoyos que reciben estudiantes con discapacidad que asisten a escuelas secundarias públicas con servicio de USAER, en comparación con los apoyos que deberían recibir, según los marcos legales y los lineamientos definidos por el Estado. Es preciso señalar que los resultados no deben generalizarse, puesto que solamente corresponden a la muestra estudiada.

En relación con el primer objetivo de la investigación, se encontró que el Estado no cubre las necesidades más importantes de los estudiantes que participaron en este estudio, pues no ayuda a las familias con becas, no ofrece apoyos tecnológicos ni ofrece soportes técnicopedagógicos, pues en las escuelas no hay suficiente material didáctico. Con respecto a los apoyos que ofrece a las escuelas, estos también son insuficientes, pues las facilidades de acceso, en el mejor de los casos, consisten en rampas. Con respecto a la planta docente, le ofrece capacitaciones improvisadas, muy teóricas y que no propician cambios en sus prácticas.

Hay varios indicadores de que el Estado mexicano no ha dimensionado la importancia de la educación inclusiva. Uno es la demora en definir cómo va a implementarse la educación inclusiva en el país. Desde 2013 se cerró el PNFEEIE y se creó el Programa Nacional para la Inclusión y Equidad Educativa (SEP, 2013), en el cual se fusionaron siete programas que antes funcionaban de manera separada, y que atendían a distintas poblaciones en condición de vulnerabilidad (por ejemplo, jornaleros migrantes, niñez en situación de rezago escolar, alumnado de telesecundaria, personas con discapacidad y estudiantes indígenas). Además de asignar un presupuesto insuficiente al programa mencionado (menos de cinco millones de dólares para el ciclo escolar 2015-2016, el cual es el total para el país) (Secretaría de Gobernación, 2015), no se han proporcionado lineamientos para su operación, por lo cual muchos profesionales desarrollan prácticas relacionadas con la integración educativa y otros con la educación inclusiva. Otro indicador es que muy poca cantidad de profesionales de la educación (inclusive de educación especial) conocen las categorías oficiales de discapacidad, lo 
cual tiene que ver con la irrelevancia de recibir ese diagnóstico, por la escasez de apoyos que se ofrece a esta población (García, 2013).

En relación con el segundo objetivo, que alude a las necesidades prácticas, metodológicas y de recursos que deben ser proporcionadas por las escuelas secundarias, se encontró que se dan algunos apoyos, aunque estos son insuficientes. Debe tomarse en cuenta que, a pesar de que cuando empezó el proceso de IE en el país había mucho rechazo a la integración educativa, actualmente hay gran aceptación (García, 2015); a esto ha contribuido la relación ER-EE, la cual hace años estaba marcada por la desconfianza (García, et al., 2003). Lo que se observó en las secundarias en que estudiaban los alumnos que participaron en la presente investigación es que dicha relación ha mejorado sustancialmente; sin embargo, necesita fortalecerse a través de las reuniones colegiadas y mediante el análisis de casos, donde ambos profesionales compartan experiencias, sugerencias y acciones con los mismos objetivos de prevención y mejora, no cuando los problemas sean más complejos y haya que intervenir de manera correctiva. Para este trabajo colaborativo entre profesionales que atienden a estudiantes se requiere de condiciones que de momento rebasan a las instituciones, pues no hay tiempos ni espacios proporcionados por el Estado para que las escuelas realicen este trabajo.

En cuanto a la capacitación y actualización de docentes, las evaluaciones que se han hecho sobre integración de los alumnos con NEE indican que los docentes conocen y están familiarizados con los principales conceptos de necesidades educativas especiales y la integración educativa (García, 2007); sin embargo, no es suficiente para lograr sensibilización a todo el colectivo, de modo que impacte al centro educativo y se refleje en sus prácticas (Jacobo, s. f.), lo cual quedó claro en las observaciones realizadas en el presente estudio, donde se vio que los docentes tienden a ser poco exigentes con los alumnos con discapacidad.

Por otro lado, el personal docente necesita estar en constante comunicación con EE para determinar si las acciones detectadas al inicio del ciclo escolar son efectivas o necesitan modificarse; es necesario que ponga atención al estudiante con discapacidad para ver sus virtudes y no solo sus limitaciones y desventajas, pues esto propicia que, lejos de ser empáticos, entorpezcan el desarrollo de sus competencias.

Los docentes lograron la identificación de los padres de familia como auxiliares y participantes de la educación de los alumnos, esto último se evidencia en la preocupación que muestran por el futuro y por el progreso académico de sus hijos. Tal como lo señalan Zaoura y Aubrey (2011), la relación padres-docentes es de vital importancia para el pleno desarrollo de competencias académicas de los estudiantes. Sin embargo, el estudiantado con discapacidad necesita una mayor y constante comunicación entre familia y docentes, que desde el inicio se acuerde el plan de intervención y las responsabilidades que cada quien asumirá.

El alumnado integrado necesita, por parte de la institución educativa a la que asiste, mayor cercanía con sus necesidades y opiniones, que sus docentes fomenten el trabajo colaborativo basado en la igualdad, respeto y valoración de las virtudes de cada quien, con el fin de integrarle y hacerle partícipe en la dinámica escolar, necesita ser visible y, además, que se le perciba con sus capacidades y virtudes, no solo con sus desventajas.

En relación con el tercer objetivo, los resultados mostraron que los apoyos que más se ofrecen a estudiantes con discapacidad en la escuela secundaria son proporcionados por EE. 
Esto se puede entender porque docentes y especialistas de $\mathrm{EE}$ tienen que entregar resultados y evidencias de su trabajo, reciben supervisión constantemente por un director o directora y la línea de comunicación con quien le supervisa es más corta que en otros servicios; además, el número de estudiantes con que trabajan es más reducido y el equipo de trabajo también es más pequeño. Aun así, hay aspectos en los que se debe mejorar, sobre todo en cuanto al impacto y relación con la escuela, pues, en general, se necesita que el servicio de USAER impacte a la institución educativa en su conjunto, que se involucre desde la planeación anual, donde aporte análisis y estrategias que garanticen acciones a favor de la inclusión.

En la presente investigación se encontró que los apoyos específicos que ofrece la USAER a estudiantes con discapacidad consisten en evaluaciones, informes, diseño del plan de intervención, conformación de expedientes con documentación detallada de la valoración, propuesta curricular adaptada (solo en el caso de [E]), actividades de seguimiento, observaciones constantes en el aula, reporte de asistencia e incidentes, como reportes o notas de desempeño por parte de sus docentes regulares, y apoyo en las evaluaciones sumativas. Para la familia, se encontró que hacen falta talleres y conferencias ofrecidas a lo largo del ciclo escolar, asesorías personales relacionadas con las evaluaciones o incidentes y orientaciones respecto a clases o actividades curriculares (solo en el caso de [E]). Para la institución en general, falta la organización de actividades generales como reuniones de consejo técnico y juntas extraordinarias.

Si bien por parte del servicio de EE se ofrece la mayoría de los apoyos, es necesario que estos se proporcionen de manera coordinada con la secundaria, con el objetivo de que el servicio de EE, como se decía antes, impacte a las instituciones en su conjunto, de tal forma que incida en las actitudes, metodologías y gestiones de los docentes, para así beneficiar a la totalidad de estudiantes, quienes necesitan que los apoyos de EE sean constantes. El servicio de EE requiere valorar de manera continua si las adecuaciones propuestas a partir de la evaluación psicopedagógica siguen siendo útiles a lo largo del ciclo escolar o si se ha superado alguna necesidad en particular y surge otra que requiera otro tipo de intervención; los cuerpos docentes de EE necesitan valorar si los apoyos gestionados para los estudiantes con discapacidad son proporcionados por parte del personal de los centros educativos con efectividad y si se corresponden al modelo inclusivo o solo se otorgan esporádicamente y como parte de la estadística a reportar en informes administrativos.

En relación con el cuarto objetivo, se encontró que las madres de familia conocen las necesidades de sus hijos y que intentan ofrecerles los apoyos que precisan, aunque esto depende de su situación económica. Las familias requieren de más orientación para fomentar la independencia de sus hijos con discapacidad y también para tomar decisiones informadas sobre su futuro académico y laboral.

\section{Conclusiones}

En México, las personas con discapacidad deberían recibir múltiples apoyos para garantizar su derecho a la educación, estipulados en la Ley General de Educación (2015), la Ley General para la Inclusión de las Personas con Discapacidad (2011), el Reglamento de la Ley General para la Inclusión de las Personas con Discapacidad (2012), el Instructivo USAER- 
CAM (SEP, 2013) y las Orientaciones generales para el funcionamiento de los servicios de educación especial (SEP, 2006). En la presente investigación, de acuerdo con los testimonios de los participantes y de las observaciones realizadas en las escuelas, se concluye que en los casos estudiados no se ofrecen, a estudiantes con discapacidad en el nivel de secundaria, todos los apoyos estipulados. Es mucho lo que se promete con respecto a lo que realmente se otorga y son todavía menores los apoyos otorgados que satisfacen realmente las necesidades del alumnado con discapacidad.

Se encontró que el Estado se limita a brindar los apoyos mínimos requeridos dentro del ambiente escolar para la generalidad de la población de educación básica. Los pocos apoyos que reciben los estudiantes con discapacidad participantes en esta investigación los proporciona el personal de educación especial (las USAER), en parte gracias a que este cumple con los criterios de evaluación y desempeño que le exige su jefatura inmediata, como conformación del expediente, evaluación psicopedagógica y orientación a padres y madres de familia. Por otro lado, estas acciones son de intervención directa e individual con estudiantes con discapacidad, prácticas que no corresponden al modelo inclusivo.

Los recursos proporcionados por parte del Estado son pocos y necesitan fortalecerse en cuanto a apoyos tecnológicos, otorgamiento de becas para actividades complementarias, servicios de salud efectivos y promoción al cambio de paradigma hacia un modelo realmente incluyente. Por parte de las secundarias públicas, el estudiantado con discapacidad necesita metodologías más flexibles que consideren sus habilidades y fortalezcan sus debilidades a través del trabajo colaborativo; necesita congruencia entre las políticas y las prácticas del centro educativo y acceso a material y recursos estimulantes para su educación. Por parte del servicio de EE, necesita constante valoración de las adecuaciones curriculares y su efectividad a lo largo del ciclo escolar, mayor vinculación con el centro educativo desde su estructura interna, fomentar la evaluación constante y acciones pertinentes que favorezcan realmente su aprendizaje y no solo la permanencia en la secundaria. Por parte de sus familiares, necesitan mayor comunicación con sus docentes de EE y ER, mayor atención y menos justificación; necesitan acompañamiento para la consolidación de habilidades que potencien sus procesos de aprendizaje.

Con frecuencia se responsabiliza del éxito de la educación, y de la educación inclusiva, a la actitud y a las prácticas pedagógicas del personal docente de educación regular, lo cual, hasta cierto punto, es correcto. Sin embargo, las familias de las personas con discapacidad, sus escuelas, sus docentes y el mismo estudiantado requieren mayores y más efectivos apoyos del Estado para que los procesos de inclusión sean exitosos, esto es, las autoridades no deben limitarse a exigir resultados sin ofrecer los apoyos que por ley deben entregar, de otra forma se convierten en las principales responsables de los fracasos de la educación en general y de la educación inclusiva en particular.

\section{Referencias}

Arape, M. y Pierre, J. (2010). Efectos de un programa psicoeducativo en el conocimiento sobre el síndrome de Asperger de docentes de educación primaria. Académica, 2(4), 71-84. Recuperado de http://dialnet.unirioja.es/buscar/documentos?querysDismax. DOCUMENTAL TODO=asperger\&inicio $=21$ 
Blanco, R. (2000). Inclusive education in Latin America. En H. Savolainen, H. Kokkala y H. Alasuutari (Eds.) Meeting Special and Diverse Educational Needs. Making Inclusive Educationa Reality [Satisfacción de las necesidades educativas especiales y diversas. Haciendo realidad la educación inclusiva]. Ministry for Foreign Affairs of Finland. Recuperado de https://www.uam.es/personal pdi/psicologia/agonzale/Asun/2006/Libros/ MeetingNeedspdf.pdf

Comisión Nacional SEP-SNTE de Carrera Magisterial. (2011). USAER y CAM. Factor aprovechamiento escolar de educación especial, (primera, segunda y tercera vertiente). Instructivo. Comisión Nacional SEP-SNTE de Carrera Magisterial. D.F., México: SEP. Recuperado de http://www.seduzac.gob.mx/carrera/documentos/ APROVECHAMIENTO ESCOLAR EDUCACION ESPECIAL 2011.pdf

Dirección de Educación Especial del Distrito Federal (2010). Memorias y actualidad de la educación especial en México. Una visión histórica de sus modelos de atención. D.F., México: Secretaría de Educación Pública. Recuperado de http://educacionespecial.sepdf. gob.mx/historia/docs/HistEduEspWeb.pdf

García, A. (2005). Concepto actual de discapacidad intelectual. Intervención Psicosocial, 14(3), 255-276.

García, I. (2007). Situación actual y retos de la integración educativa en México. En S. Romero y I. García (Coords.), Estudios sobre la educación básica y la educación especial en México. San Luis Potosí, México: Universidad Autónoma de San Luis Potosí.

García, I. (2009). Educación inclusiva en Latinoamérica y el Caribe. Diagnóstico actual y desafíos para el futuro. San Luis Potosí: México: Banco Mundial-Universidad Autónoma de San Luis Potosí. México.

García, I. (2013). La educación especial decara a la educación inclusiva. Revista Latinoamericana de Educación Inclusiva, 7 (2), 15-23.

García, I. (2015). Integración educativa o educación inclusiva, ¿qué conviene a México? Cátedra CUMEX de Psicología. En A. García y O. Cruz, Los retos de la psicología en la sociedad contemporánea. Tuxtla Guitiérrez Chiapas, México: Universidad de Ciencias y Artes de Chiapas.

García, I. y Romero, S. (s. f.). Protocolos de observación y entrevista para la evaluación de prácticas inclusivas y calidad de la educación. Manuscrito sin publicar.

García, I., Escalante, I., Escandón, M. C., Fernández, L. G., Mustri, A. y Puga, I. (2000). La integración educativa en el aula regular. Principios, finalidades y estrategias. D.F., México: SEP-Fondo Mixto México-España. 
García, I., Escalante, I., Escandón, M. C., Fernández, L. G., Mustri, A. y Puga, I. (2003). Proyecto de investigación e innovación integración educativa. Informe parcial de investigación (Ciclos escolares 1997-1998). En Integración educativa. 1996-2002 Informe final. [CD] Secretaría de Educación Pública, Subsecretaría de Educación Básica y Normal, Fondo Mixto de Cooperación Técnica y Científica y Cooperación Española.

García, I., Romero, S., Motilla, K. y Zapata, C. (2009). La reforma fallida de los centros de atención múltiple en México. Actualidades Investigativas en Educación, 9(2), 1-21. Recuperado de http://revista.inie.ucr.ac.cr/index.php/aie/article/viewFile/325/324

Gobierno Constitucional de los Estados Unidos Mexicanos. (Martes 19 de mayo de 1992). Acuerdo Nacional para la Modernización de la Educación Básica. Diario Oficial de la Federación. Recuperado de http://www.sep.gob.mx/work/models/sep1/Resource/ b490561c-5c33-4254-ad1c-aad33765928a/07104.pdf

Gobierno Constitucional de los Estados Unidos Mexicanos. (Publicada 13 de julio de 1993 y reformada 17 de diciembre del 2015). Ley General de Educación. Diario Oficial de la Federación. Recuperado de https://www.sep.gob.mx/work/models/sep1/ Resource/558c2c24-0b12-4676-ad90-8ab78086b184/ley general educacion.pdf

Gobierno Constitucional de los Estados Unidos Mexicanos. (30 de mayo de 2011). Ley General para la Inclusión de las Personas con Discapacidad. Diario Oficial de la Federación, Recuperado de http://www.dof.gob.mx/nota detalle.php?codigo=5191516\&fecha=30/05/2011

Gobierno Constitucional de los Estados Unidos Mexicanos. (30 de noviembre del 2012). Reglamento de la Ley General para la Inclusión de las Personas con Discapacidad. Diario Oficial de la Federación. Recuperado de http://normatecainterna.sep.gob. mx/work/models/normateca/Resource/229/1/images/reglamento ley general inclusion personas discapacidad.pdf

Gobierno Constitucional de los Estados Unidos Mexicanos. (2015). Acuerdo número 24/12/14 por el que se emiten las Reglas de Operación del Programa para la Inclusión y la Equidad Educativa para el ejercicio fiscal 2015. Diario Oficial de la Federación. Recuperado de http://dof.gob.mx/nota detalle.php?codigo=5377499\&fecha=27/12/2014

Jacobo, Z. (s. f.). Las prácticas de integración educativa: Alcances, preguntas y propuestas. Recuperado de http://www.comie.org.mx/congreso/memoriaelectronica/v09/ponencias/ at14/PRE1178943648.pdf

Jacobo, Z., Ortiz, A., Gasca, J., Alvarado, J. A., Ehrenberg, M., Melgar, J. y Villa, M. A. (2005). Informe final. Evaluación externa del Programa Nacional de Fortalecimiento de la Educación Especial y de la Integración Educativa. México. 
Mares, A. y Ito, E. (2005). Integración educativa. Perspectiva desde los actores sociales encargardos de realizarla. Revista Mexicana de Investigación Educativa, 10(26), 903-930. Recuperado de http://www.comie.org.mx/documentos/rmie/v10/n026/pdf/ rmiev10n26scC00n02es.pdf

Metz, K. (2011). Taller de investigación cualitativa-observación en aula. Universidad de Arizona. E.U.A.

Muñoz, A. y Portillo, R. (2007). Evaluación psicopedagógica de la discapacidad intelectual y del retraso límite: Elementos y modos de evaluación. Apuntes de Psicología, 25(2), 111128. Recuperado de http://dialnet.unirioja.es/servlet/articulo?codigo $=2350680$

Organización de las Naciones Unidas. (2006). Convención sobre los Derechos de las Personas con Discapacidad. New York. Recuperado de http://www.educacionespecial.sep.gob.mx/ pdf/materialesdoctos/convencion.pdf

Romero, S. y García, I. (2013). Educación especial en México. Desafíos de la educación inclusiva. Revista Latinoamericana de Educación Inclusiva, 7(2), 77-91. Recuperado de http://www.rinace.net/rlei/numeros/vol7-num2/art5.pdf

Secretaría de Educación Pública (SEP). (2002). Programa Nacional para el Fortalecimiento de la Educación Especial y la Integración Educativa. D.F., México: SEP. Recuperado de http://www.educacionespecial.sep.gob.mx/pdf/publicaciones/ProgNal.pdf

SEP. (2006). Orientaciones generales para el funcionamiento de los servicios de educación especial. D.F., México: SEP. Recuperado de http://www.educacionespecial.sep.gob.mx/ pdf/publicaciones/libromorado.pdf

SEP. (2009). Modelos de atención educativa de los servicios de la dirección de educación especial en el Distrito Federal. D.F., México: SEP. Recuperado de http://educacionespecial. sepdf.gob.mx/documentos/MASEE2011.pdf

SEP. (2013). Acuerdo número 711 por el que se emiten las Reglas de Operación del Programa para la Inclusión y la Equidad Educativa. D.F., México: SEP. Recuperado de http://dof. gob.mx/nota detalle.php?codigo $=5328358 \&$ fecha $=28 / 12 / 2013$

UNESCO, UNICEF y Fundación Hineni (2003). Cada escuela es un mundo, un mundo de diversidad. Experiencias de integración educativa. Santiago de Chile: UNESCO, UNICEF, Fundación Hineni.

Zaoura, A. y Aubrey, C. (2011). Home-schools relationships in primary schools-parent's perspectives [Perspectiva de los padres en la relación casa- escuela primaria]. Interntional Journal Parents in Education, 5(2), 12-24. Recuperado de http://www. ernape.net/ejournal/index.php/IJPE/article/viewFile/161/108 